Liver Transplantation

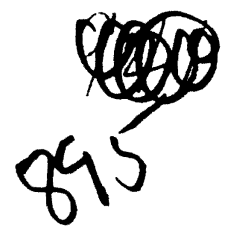

\title{
Orthotopic Liver Transplantation for Fulminant and Subacute Hepatic Failure
}

\author{
Andrei C. Stieber, M.D., Giovanni Ambrosino, M.D., \\ David Van Thiel, M.D., Shunzaburo Iuatsuki, M.D., \\ and Thomas E. Starzl, M.D., Ph.D.
}

Fulminant or subacute hepatic failure (FHF/SAF) are characterized by massive necrosis of hepatocytes caused by any of a wide variety of hepatic insults (viral infection, chemicals, metabolic disorders, etc.). The condition is defined as true fulminant failure when it occurs within 8 weeks of the onset of the symptoms and subacute when the liver failure becomes evident sometime between the eighth and the twentieth week after the onset of the symptoms of liver disease. True FHF presents with progressive deterioration of hepatic function, leading to deepening jaundice, rapid onset and progression of hepatic encephalopathy, "foetor hepaticus," edema, ascites, severe coagulation disturbances and, in the later stages, hypoglycemia. hepatorenal syndrome, sepsis, acidosis, multiorgan failure, and eventually death. FHF is a disease syndrome with an extremely high degree of morbidity and mortality. The mortality is age- and etiologydependent and averages 80 per cent when Stage IV coma has been reached, despite intensive medical treatment. ${ }^{-\rightarrow}$ In adults who reach stage IV coma, the mortality is 95 per cent or greater. SAF presents with a slower progression, but with a similar outcome in most of the cases. While liver transplantation (ortho- or heterotopic) has always been a tempting alternative to intensive medical therapy for this condition, it is only recently that whole organ liver replacement has achieved a success rate that justifies its use for $\mathrm{FHF} / \mathrm{SAF}{ }^{3-s}$

Departments of Surgery and Medicine, University of Pittsburgh School of Medicine. Pittsburgh. Pennsylvania

This work was supported in part by the following grants: 2R01 AA04427-07 ADAMHA. awarded by the National Institute on Alcohol Abuse and Alcoholism: 2R01 DK32556-05 NI, awarded by the National Institute of Diabetes and Digestive and Kidney Diseases; 5R01 A.106601-03 NIH, awarded by the National Institute on Alcohol Abuse and Alcoholism 


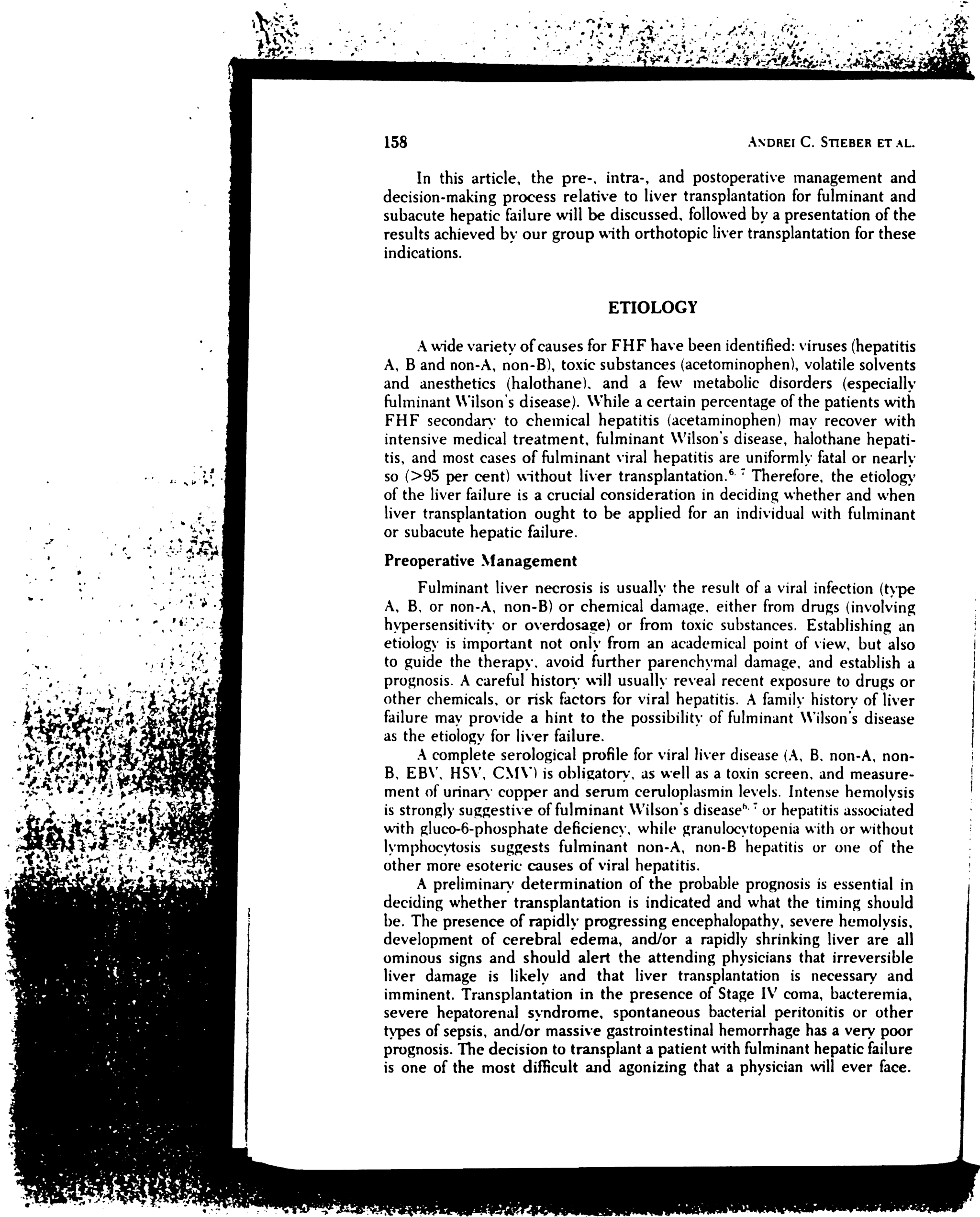




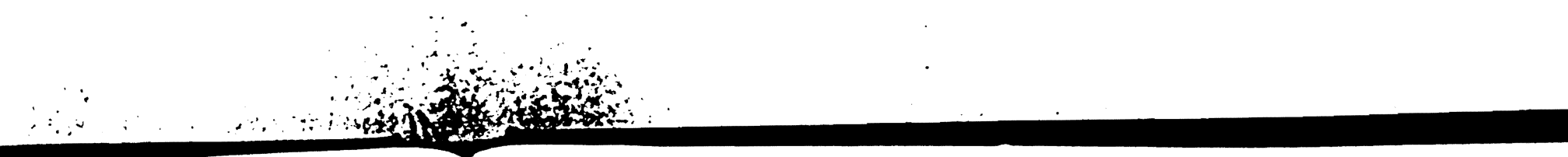

ANDREI C. STIEBER ET Al.

postoperative management and ransplantation for fulminant and followed by a presentation of the pic liver transplantation for these

:Y

been identified: viruses (hepatitis acetominophen), volatile solvents metabolic disorders (especially in percentage of the patients with (etaminophen) may recover with lilson's disease, halothane hepatititis are uniformly fatal or nearly itation. ${ }^{6 .}$ : Therefore, the etiology on in deciding whether and when I for an individual with fulminant

the result of a viral infection (type nage, either from drugs (involving toxic substances. Establishing an kademical point of view. but also (nchyinal damage, and establish a reveal recent exposure to drugs or hepatitis. A family history of liver bility of fulminant Wilson's disease

ral liver disease (A, B. non-A. nonvell as a toxin screen, and measureloplasmin levels. Intense hemolysis in' disease in $^{7}$ or hepatitis associated i. granulocytopenia with or without A. non-B hepatitis or one of the ititis.

11. probable prognosis is essential in dicated and what the timing should : encephalopathy, severe hemolysis, or a rapidly shrinking liver are all tending physicians that irreversible $r$ transplantation is necessary and 'nce of Stage IV coma, bacteremia, ncous bacterial peritonitis or other lestinal hemorrhage has a very poor patient with fulminant hepatic failure ang that a physician will ever face.

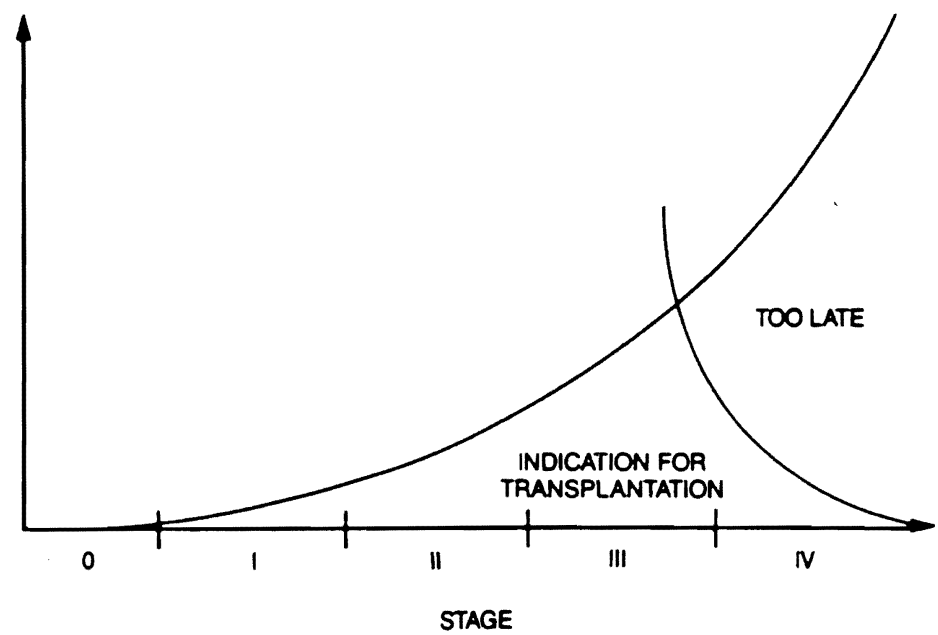

Figure 1. The indication for OLTx increases until the early stage IV' coma. then decreases rapidly.

The favorable "window" for transplantation may be extremely brief, and temporizing may adversely affect the patient's chance for survival (Fig. 1). Generally, rapid deepening of the hepatic coma, a steady prolongation of the prothrombin time (unresponsive to infusion of fresh frozen plasma), the development of the hepatorenal sundrome, hypoglycemia. and uncorrectable metabolic acidosis are signs of impending death that require urgent transplantation. Frequent asessment of the patient's condition, as often as hourly, is necessary in order to be able to make a proper decision. As a result of the considerable improvement in liver transplantation results experienced during the past 5 or 6 years, the decision to proceed with liver transplantation in cases of FHF or SAF (also called "late-onset hepatic failure") is less difficult than previously.

It is essential to place the patients with $\mathrm{FHF}$ on the urgent transplant list as soon as they are admitted to hospital. Age. blood type, height, and weight must be obtained to permit a good donor-recipient match, if possible: matching for the blood type is desirable, though not essential, while a good size match is highly indicated for technical reasons. The clinical situation of the patient must be assessed every time a potential donor organ becomes available, and a liver transplant should be performed if the patient's condition is thought to be irreversible without organ replacement and if the donor is suitable. If the situation is particularly desperate, a liver that is of a different blood type and/or size can be used. even if such grafts result in less than ideal transplant outcomes. A brief, but intensive work-up should be performed, including a determination of the blood group, sonography to assess the patency of the portal and suprahepatic veins, viral hepatitis and toxic screens, urine copper excretion, and serum ceruloplasmin level. All potential infection should be avoided and any existing infection must be treated early and aggressively. The coagulation status should be corrected as much as possible with fresh frozen plasma (FFP) infusions and the administration of exogenous vitamin $K$, 


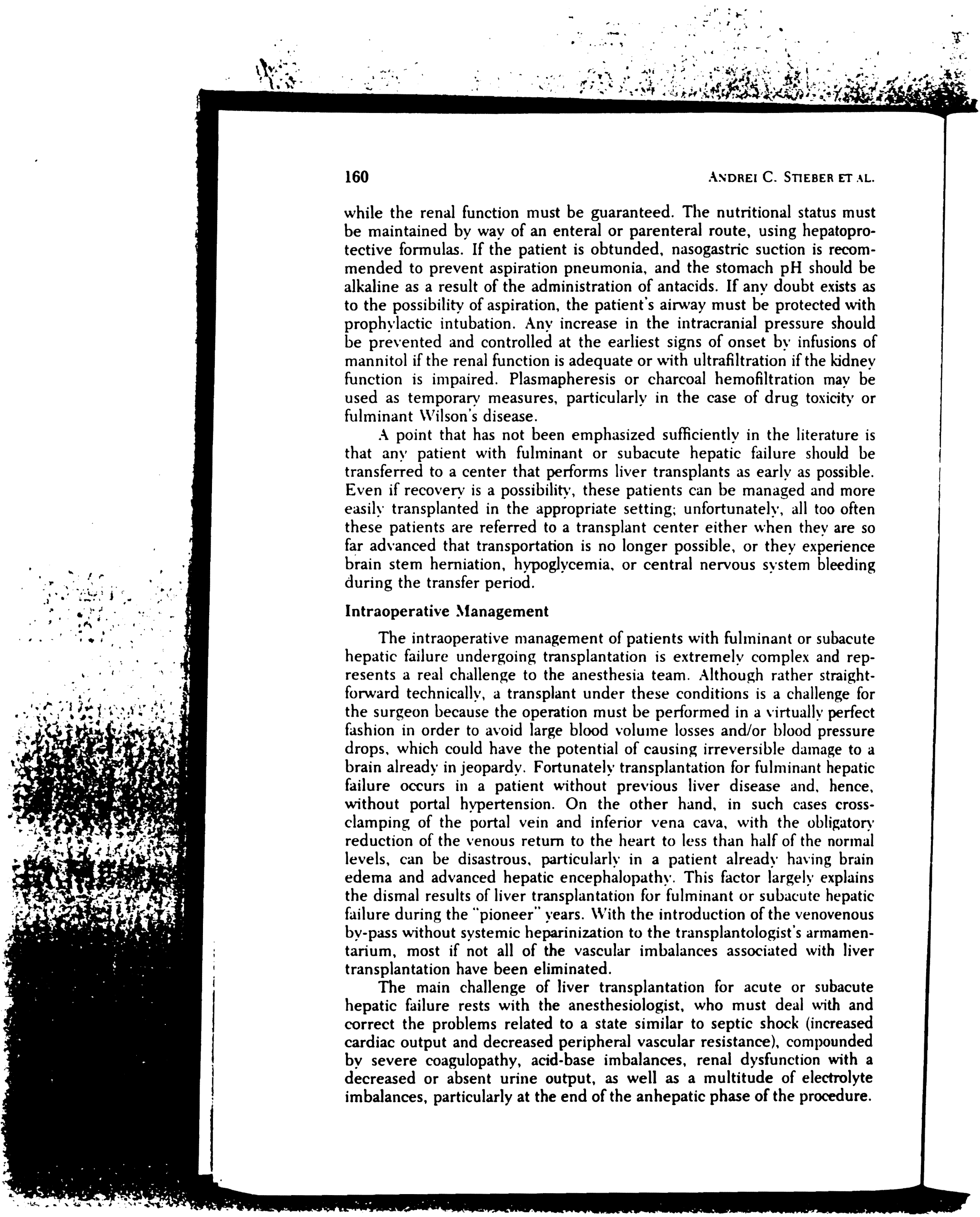




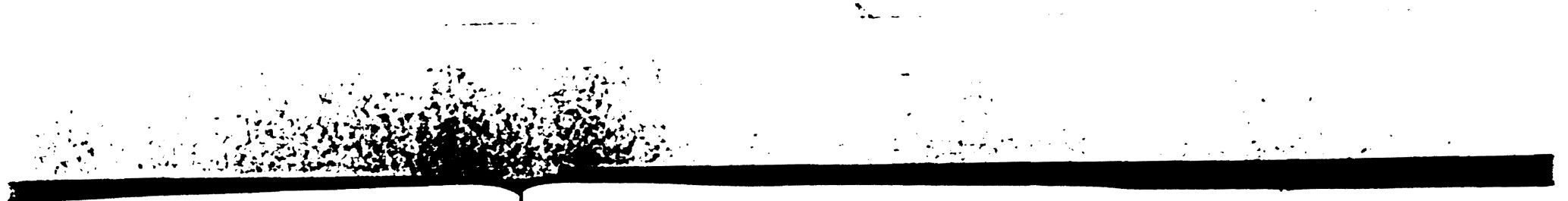

ranteed. The nutritional status must $r$ parenteral route, using hepatoprounded, nasogastric suction is recominia, and the stomach $\mathrm{pH}$ should be on of antacids. If any doubt exists as ient's ainway must be protected with in the intracranial pressure should arliest signs of onset by infusions of ste or with ultrafiltration if the kidney or charcoal hemofiltration may be larly in the case of drug toxicity or

usized sufficiently in the literature is subacute hepatic failure should be liver transplants as early as possible. e patients can be managed and more setting; unfortunately, all too often plant center either when they are so , longer possible, or they experience or central nervous system bleeding

of patients with fulminant or subacute :ation is extremely complex and rephesia team. Although rather straightler these conditions is a challenge for ust be performed in a virtually perfect volume losses and/or blood pressure al of causing irreversible damage to a 1. transplantation for fulminant hepatic previous liver disease and, hence, ne other hand, in such cases crossferior vena cava, with the obligatory t. heart to less than half of the normal Is in a patient already having brain !alopathy. This factor largely explains ition for fulminant or subacute hepatic ith the introduction of the venovenous ion to the transplantologist's armamenular imbalances associated with liver 1.

:ransplantation for acute or subacute thesiologist, who must deal with and tate similar to septic shock (increased leral vascular resistance), compounded imbalances, renal dysfunction with a as well as a multitude of electrolyte the anhepatic phase of the procedure.
The anesthesia team is, therefore, faced with an extremely complex situation that requires constant attention throughout the procedure. The correction of fluid and electolyte imbalances is done in a continuous manner; the coagulation is modulated and enhanced by the use of FFP, platelet and cryoprecipitate infusion, as well as by administration of epsilon-aminocaproic acid when fibrinolysis occurs. Frequent thromboelastogram (TEG) monitoring is required to accomplish these goals. Intraoperative EEG monitoring is recommended, since the presence of seizure activity cannot be ascertained othenwise under general anesthesia. The use of vasopressors for the control of hypotension during the operative procedure has to be extremely cautious, since they can damage the allograft by decreasing the splanchnic blood flow.

\section{Postoperative Management}

The postoperative management of individuals transplanted for FHF or $S A F$ is different from that utilized in other patients who have been transplanted, because these FHF/SAF patients may still have residual renal failure, requiring adjustment of their cyclosporine (CsA) doses and as a result, may need the addition of other immunosuppressive agents to compensate for the lower CsA level (azathioprine, antithymocyte globulin [ATG], or monoclonal antibody preparation [OKT3]) and/or hemodialysis, until the renal function resumes. In such cases, the liver function must be monitored more carefully than usual, as primary nonfunction of the allograft tends to be more lethal in patients whose brain is already impaired as a result of preexisting encephalopathy; if the allograft function during the early postoperative period is not excellent, retransplantation may be performed as an emergency to limit further patient deterioration.

\section{RESULTS OF LNER TRANSPLANTATION FOR FULMINANT AND SUBACUTE HEPATIC FAILURE}

Our experience includes 40 patients who underwent liver transplantation for fulminant and subacute hepatic failure in a series of 1,170 patients transplanted between .March, 1963 and July 20, 1987. Of these 40 patients, 17 ( 42.5 per cent) were male and $23(5, .5$ per cent were female. Their ages ranged from 4 to 62 years (overall mean 21.75), with a range of 4 to 62 years (mean 21.41) for males and a range of 6 to 5 - years (mean 22.00) for females. Eight patients (20 per cent) had hepatitis type $B$ ( 3 male and 5 female), fifteen ( 37.5 per cent) had hepatitis type non- $A$, non-B $(6$ male, 9 female), six (15 per cent) had fulminant chemical toxicity ( 4 male, 2 female), ten ( 25 per cent) had fulminant Wilson's disease ( 4 male, 6 female) and one female had FHF of unknown etiology (possibly Reye's syndrome) (Fig. 2). Thirty (75 per cent) presented with acute hepatic failure (14 male and 16 female) and ten ( 25 per cent) had subacute hepatic failure $(3$ male and 7 female). Six (15 per cent) had very mild or no encephalopathy (2 male, 4 female), eight (20 per cent) had stage I hepatic coma ( 4 male and 4 female), five (12.5 per cent) had stage II hepatic coma ( 2 male and 3 female), seven (17.5 per cent) had stage III hepatic coma ( 2 male, 5 female) 
162

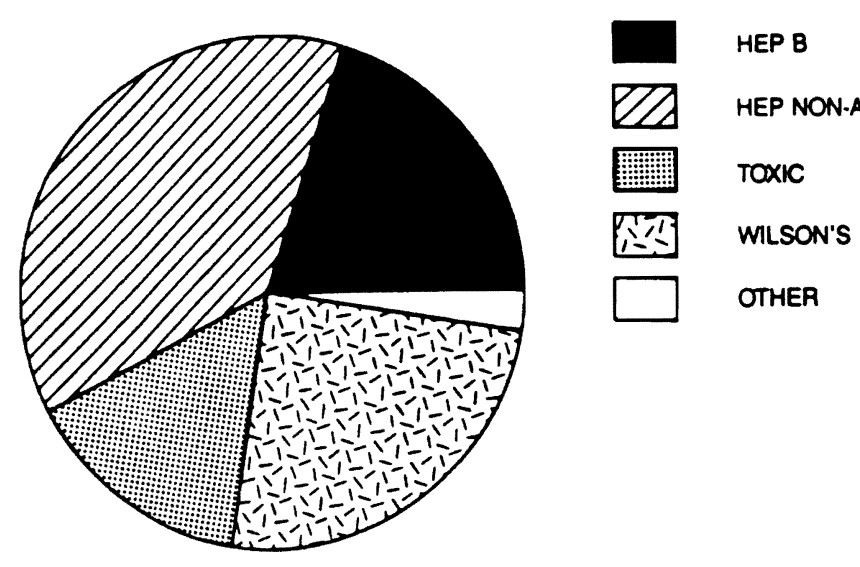

Figure 2. Fulminant and subacute hepatic failure diagnoses.

and fourteen ( 35 per cent) had stage III/IN or IV' coma ( 6 male, 8 female). The record was incomplete in one case and the degree of coma could not be determined retrospectively (Fig. 3). The interval from the onset of clinical disease to the time of transplantation ranged from 0 to 25 weeks (mean 5.37), the total pretransplant hospital time ranged from less than 1 to 40 davs (mean 10.6), the pretransplant $\mathrm{ICC}$ time ranged from 0 to 6 days (mean 2.0), while the time spent on a respirator before transplantation ranged from 0 to 5 davs (mean 0.95 ). 23 patients $(5 \pi .5$ per cent) survived ( 10 male and 13 female), while 17 ( $\$ 2.5$ per cent) died $(7$ male and 10 female) (Fig. 4). There was no significant statistical difference in the total pretransplant, hospital and ICL time between the survivor and nonsurvivor groups. A trend toward a longer time spent on the respirator prior to transplantation was evident in nonsurvivors $(0.76$ days for survivors versus 1.18 days for nonsurvivors), although the number of patients in this group was too small to achieve a statistical significance. Although in previous studies" a significant negative impact of retransplantation on survival was reported, we did not observe such a relationship in this series. In fact, in

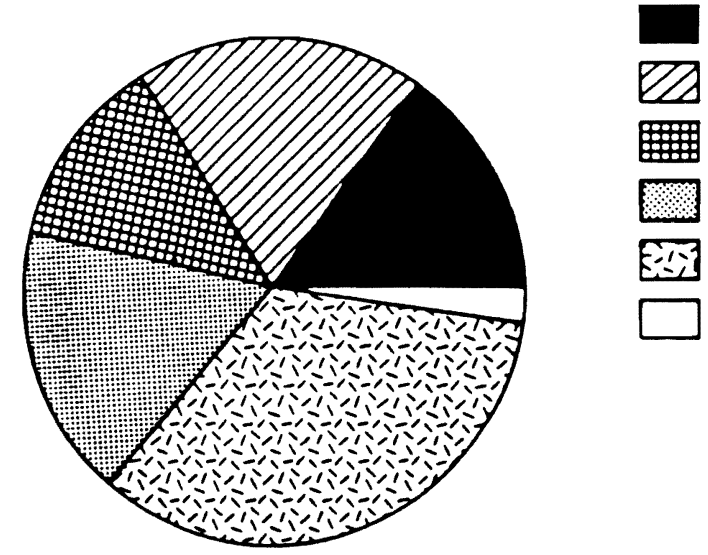

VERY MILD OR NONE

GRADE I

GRADE II

GRADE III

GRADE IIUTV

UNKNOWN

Figure 3. Fulminant and subacute hepatic failure: Degree of coma, number of patients. 


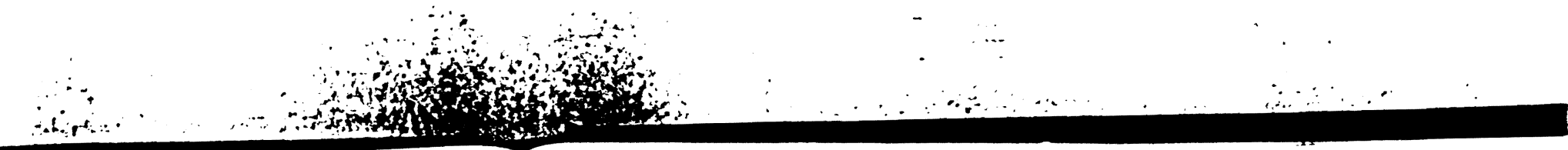

ANDREI C. STIEBER ET AL.

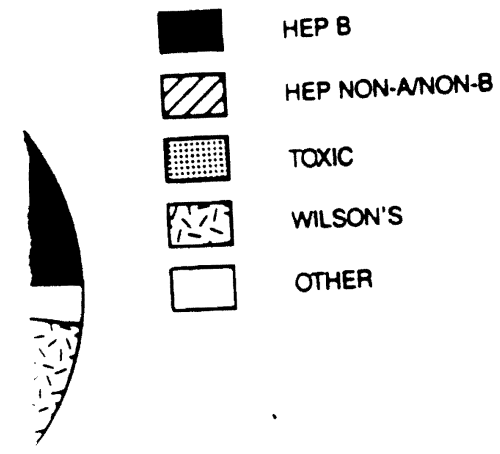

hacute hepatic failure diagnoses.

III/IV or IV coma (6 male, 8 female). ase and the degree of coma could not 3). The interval from the onset of plantation ranged from 0 to 25 weeks hospital time ranged from less than 1 nsplant ICC time ranged from 0 to 6 it on a respirator before transplantation i). 23 patients (57.5 per cent) survived ( $\$ 2.5$ per cent) died $(7$ male and 10 ificant statistical difference in the total - between the survivor and nonsurvivor time spent on the respirator prior to univors 0.76 days for survivors versus h) the number of patients in this group cal significance. Although in previous ict of retransplantation on survival was a relationship in this series. In fact, in

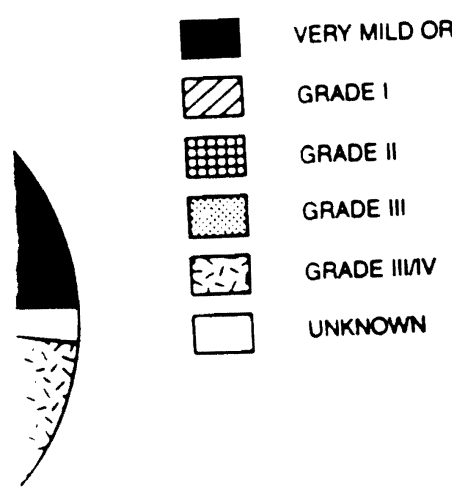

IC failure: Degree of coma, number of patients.

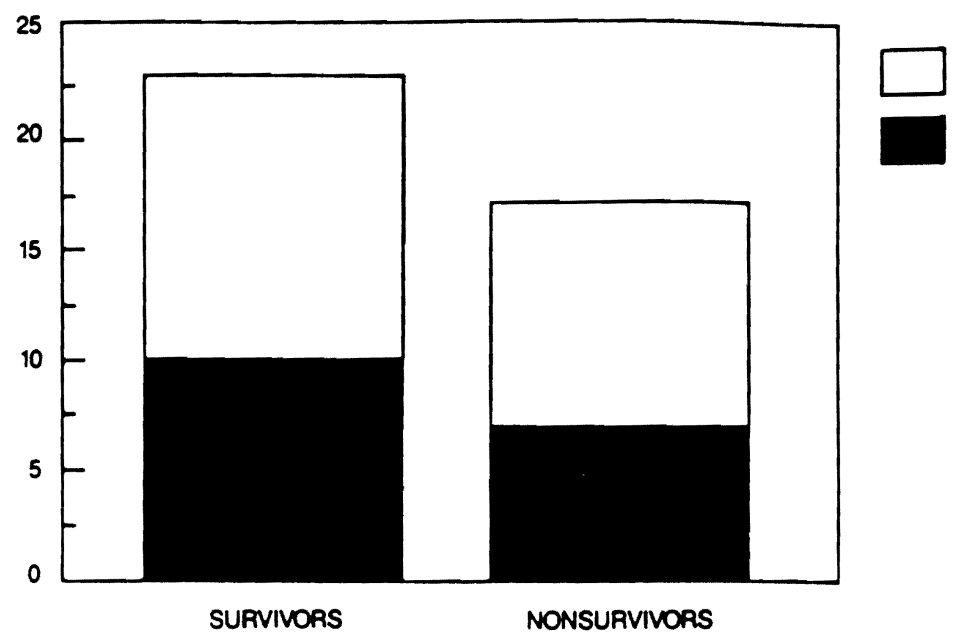

FEMALES MALES

Figure 4. Fulminant and subacute hepatic failure: Survival by sex.

this series, the only patient who received four consecutive liver transplants survived. On the other hand, it appears that the need for very early retransplantation may adversely affect survival.

Of the 40 patients in our series, 22 ( 55 per cent) had a good neurological outcome, meaning a complete recovery of their neuropsychic performance, without any evidence of neurological sequellae; 2 patients ( 5 per cent) had a fair neurological outcome with persistence of some sequellae; finally, 16 patients ( 40 per cent) had a poor neurological outcome (Fig. 5). This latter group included all the nonsurvivors who never awoke after the transplant operation, as well as 2 patients who survived for at least 6 months or longer but never regained sufficient neuropsychic capacity to function independently. There was no statistical difference in the total pretransplant, hospital, and ICU time between the patients with a good and a poor neurological outcome. There was a trend toward a difference between the two groups

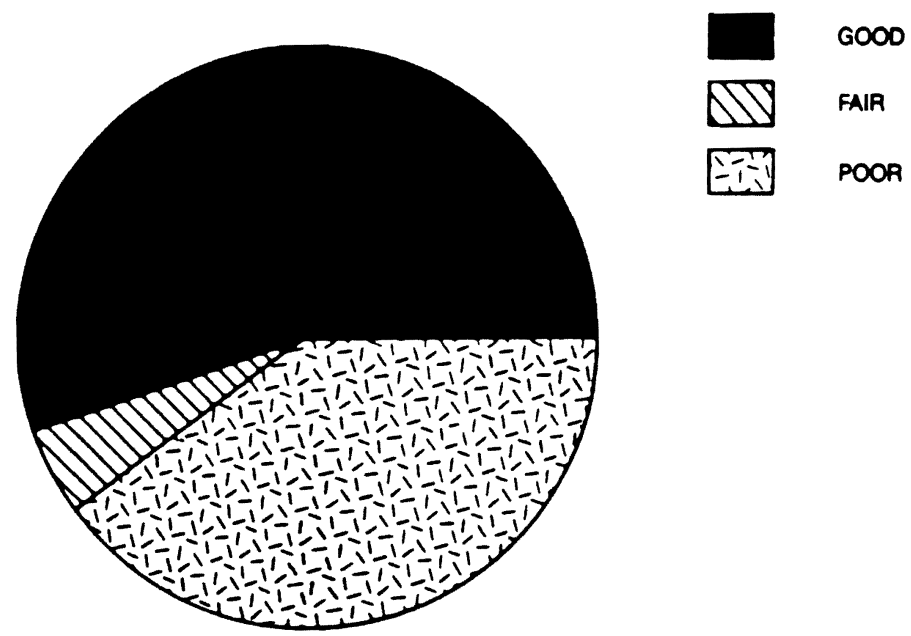

Figure 5. Fulminant hepatic failure: Neurological outcome. 
when the time spent in the ICU and on a respirator preoperatively was considered (1.77 day's for a good neuropsychiatric outcome versus 2.25 for a poor outcome and 0.65 days vs. 1.37 day's respectively). The number of patients in each group, however, was too low to achieve a statistical difference. There was a notable difference between good and poor neurological outcomes when the degree of coma was analyzed: 9 out of 16 patients with a poor outcome ( 56.25 per cent) had stage III/IV or IV coma, while only 4 out of 22 (18 per cent) patients with a good neurological outcome had advanced coma grade. When the patients who required retransplantation were analyzed, the most lethal of the causes for retransplantation was found to be the primary nonfunction of the allograft; because only one out of five ( 20 per cent) survived, this is consistent with our previous findings. ${ }^{s}$

\section{DISCUSSION}

Considering the dismal prognosis of fulminant and subacute hepatic failure, it would seem to be a prime indication for liver replacement. intil just a few years ago, this was not feasible in practice, as the results with liver transplantation, in general, were rather poor, and to this the additional handicaps associated with FHF would have to be added. Since the introduction of Cs.A, the results with liver transplantation have improved enormously and with the greater availability of donors, liver replacement for FHF has become a reasonable proposition. All other methods of temporary hepatic support utilized have only provided additional time during which a donor organ can be actively sought, but none of these represent a valid definitive alternative to liver transplantation. These methods should be used routinely whenever possible during the pretransplant period to slow down the speed of the hepatic failure and to allow the patient to be transplanted while still being in the best possible condition. Notable in this respect are the use of activated charcoal hemofiltration, plasmapheresis, and the use of prostacyclin infusion to prevent platelet aggregation. ${ }^{1.2}$

It is of some interest that only one of the cases of FHF caused by hepatitis B virus infection, who received $100 \mathrm{ml}$ of hyperimmune globulin during the operation, has converted to antigen negative/antibody positive; one other patient, the first perioperative survivor of a transplant for FHF performed in 1974, appeared to be hepatitis B negative by RIA after the transplant procedure, but the subsequent records for this patient are incomplete and he died 3 months later of complications of the transplant unrelated to his original disease. One patient died on the operating table. All the others (62.5 per cent) have continued to be serologically positive after the transplant, and all but one of these had recurrence of the disease (proven by biopsy). They are all stable and well now, although with active low-grade disease, 8 months to 3 years after their transplant. There is no doubt that most of the patients with FHF regardless of etiology have a survival of only 20 per cent or less with even the most intensive medical treatment, while transplantation offers immediate survival of at least 55 per cent. 


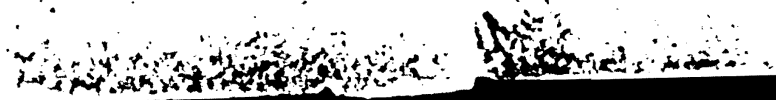

IDREI C. StTEBER ET Al.

or preoperatively was itcome versus 2.25 for ively). The number of o achieve a statistical good and poor neuroed: 9 out of 16 patients IV or IV coma, while I neurological outcome required retransplanta$r$ retransplantation was : because only one out our previous findings.

$t$ and subacute hepatic iver replacement. Intil ice. as the results with nd to this the additional added. Since the introntation have improved nors, liver replacement tll other methods of rovided additional time ght, but none of these transplantation. These ble during the pretransfailure and to allow the best possible condition. charcual hemofiltration, sion to prevent platelet

ases of FHF caused by if hiperimmune globulin gative/antibody positive; of a transplant for FHF cLative by RIA after the rds for this patient are ications of the transplant $\mathrm{d}$ on the operating table. be serologically positive recurrence of the disease low, although with active ir transplant. There is no rdless of etiology have a c most intensive medical survival of at least 55 per
There is little doubt, from our data as well as that of others, that liver transplantation for FHF/SAF is not only justified, but indicated and that, with continuous improvement of the technique, transplantation should be offered as an alternative earlier than ever before and in some cases even before spontaneous recovery can be ruled out completely.

\section{REFERENCES}

1. EASL: Randomised trial of steroid therapv in acute liver failure. Gut 20:620, 1979

2. Gimson AES. Braude S, Mellon PJ, et al: Earlier charcoal haemoperfusion in fulminant hepatic failure. Lancet September 25:681. 1982

3. Iwatsuki S. Esquivel CO. Gordon RD. et al: Liver transplantation for fulminant hepatic failure. Semin Liver Dis 5:325, 1985

4. O'Grady JJ. Williams R. Calne RY: Transplantation in fulminant hepatic failure. Lancet November 22:1227, 1986

5. Pichlmayr R. Broelsch CE. Tidow G. et al: Liver transplantation and impending hepatic failure. In Brunner G. Schmidt FW teds): Artificial Liver Support. Berlin. Heidelberg. New York. Springer Verlag. 1980. pp 322-32\%

6. Rakela J. Kurtz. SB. McCarthy JT, et al: Fulminant Wilson s disease treated with postdilution hemofiltration and orthotopic liver transplantation. Gastroenterology 90:2004. 1986

7. Sokol RJ. Francis PD. Gold SH, et al: Orthotopic liver transplantation for acute fulminant Wilson's disease. $107(t): 549,1985$

S. Starzl TE. Iwatsuki S. Shaw BW Jr. et al: Liver transplantation in the crclosporine era. Prog Allergy 38:366, 1986

Transplantation Surgery Office

Falk 5C. L'niversity' of Pittsburgh School of Medicine

36015 th Avenue

Pittsburgh. Pennswiliania 15261 